MATEC Web of Conferences 22,03009 (2015)

DOI: $10.1051 /$ matec conf/ 20152203009

(C) Owned by the authors, published by EDP Sciences, 2015

\title{
A Study on the Intelligent Analysis and Pre-warning Platform of Power Grid Video Surveillance Based on "the Integration of Regulation and Control"
}

\author{
Lijun Feng, Junliu Zhang, Peilin Chen, Mingdi Li, Xingquan Zhao \& Yang Liu \\ Power Dispatching Control Center, State Grid Shanxi Electric Power Company, Taiyuan, Shanxi, China
}

\begin{abstract}
In order to strengthen the centralized management and control on the work of substations in all aspects and increase the real-time surveillance and safety level of unattended substations, this paper carries out a study on the intelligent analysis and pre-warning platform of power grid video surveillance based on "the integration of regulation and control". With the design idea of combining centralization and distribution, this platform screens and analyzes a large amount of videos intelligently through the target characteristic detection method based on vision and the means of pattern classification, realizing accurate warnings of the work of unattended substations.
\end{abstract}

Keywords: video surveillance; image processing; image recognition; SVM classifier

\section{INTRODUCTION}

With the development of the network technology, the video technology, power system protection and the automatic control, the automatic operation level of substations increases unceasingly. The intelligent system provides substations with more reliable and safe operational environment so as to reduce manual operating accidents greatly.

Controllers will face a huge challenge in the video inspection of supervisee stations due to the explosion in the number of substations and surveillance videos. The ability of inspecting video images of each supervisee station effectively and the quality of related inspection work will be decreased because of the information overload ${ }^{[1]}$. An intelligent remote vision analysis pre-warning system ${ }^{[2-3]}$ needs to be studied, which provides services for monitoring controllers by studying the post processing and analysis with existing video network and data of the video surveillance information platform. Monitoring controllers are assisted in mastering such situations of the supervisee station as environmental condition, equipment operation and civilized production, strengthening the surveillance on severe conditions that pose a threat to safety production like bad weather, fire hazard, equipment explosion, equipment surface failure, equipment stealing and malicious destruction, conducting crucial and accurate detections on unusual conditions, so that scientific decisions and real-time responses can be made out on incidents quickly and the real-time monitoring and safety level of the unattended substation can be improved effectively. This paper mainly studies the video surveillance system based on the platform of the integration of regulation and control ${ }^{[4]}$.

\section{NECESSITY AND DESIGN SCHEME OF THE PLATFORM CONSTRUCTION}

The integration of regulation and control refers to the integrated management on regulation and control, which not only is convenient for the unified management of the dispatching center but also avoids the waste of resources by saving part of the large management cost ${ }^{[5]}$. Besides, the intelligent video surveillance system constructs a window of man-machine conversation for the integrated regulation and control platform. The advanced $\mathrm{B} / \mathrm{S}$ architecture monitoring and control system is now adopted so that managers of the dispatching center can know the operating status of substation equipment and the external environment change of the substation through a browser.

The research objective of this paper is to conduct automatic inspections on substations and know the real-time status of substations through video surveillance devices. Omissions of the inspection work can be effectively avoided as well ${ }^{[5]}$. Meanwhile, with the intelligent video source data decoded and output by the intelligence algorithm and the analysis on the intelligent video source data, unusual conditions of supervisees can be located accurately, analyzed intelligently and pre-warned in time. This paper aims at realizing the intelligent monitoring on the real-time operation of the power grid, improving the monitoring efficiency of power grid operation and ensuring the safe and stable operation of power equipment. The overall design structure of the platform is shown in Figure 1:

The design idea of the platform is to combine centralization and distribution ${ }^{[6]}$. The centralized management is combined with the processing and distribution of the algorithm through the front end of the acquisition simulation. 
MATEC Web of Conferences

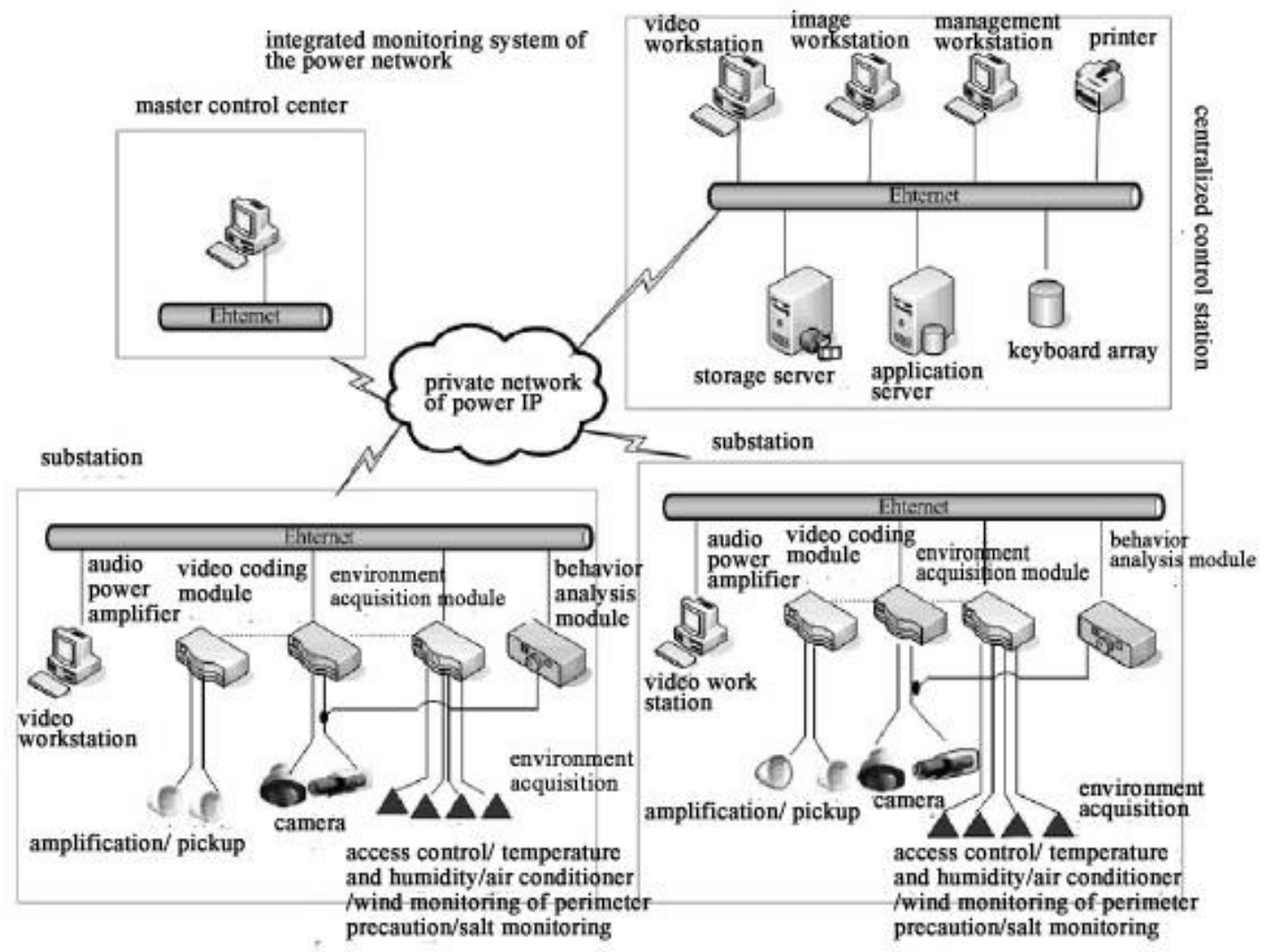

Figure 1. The overall design structure of the video surveillance of unattended substations

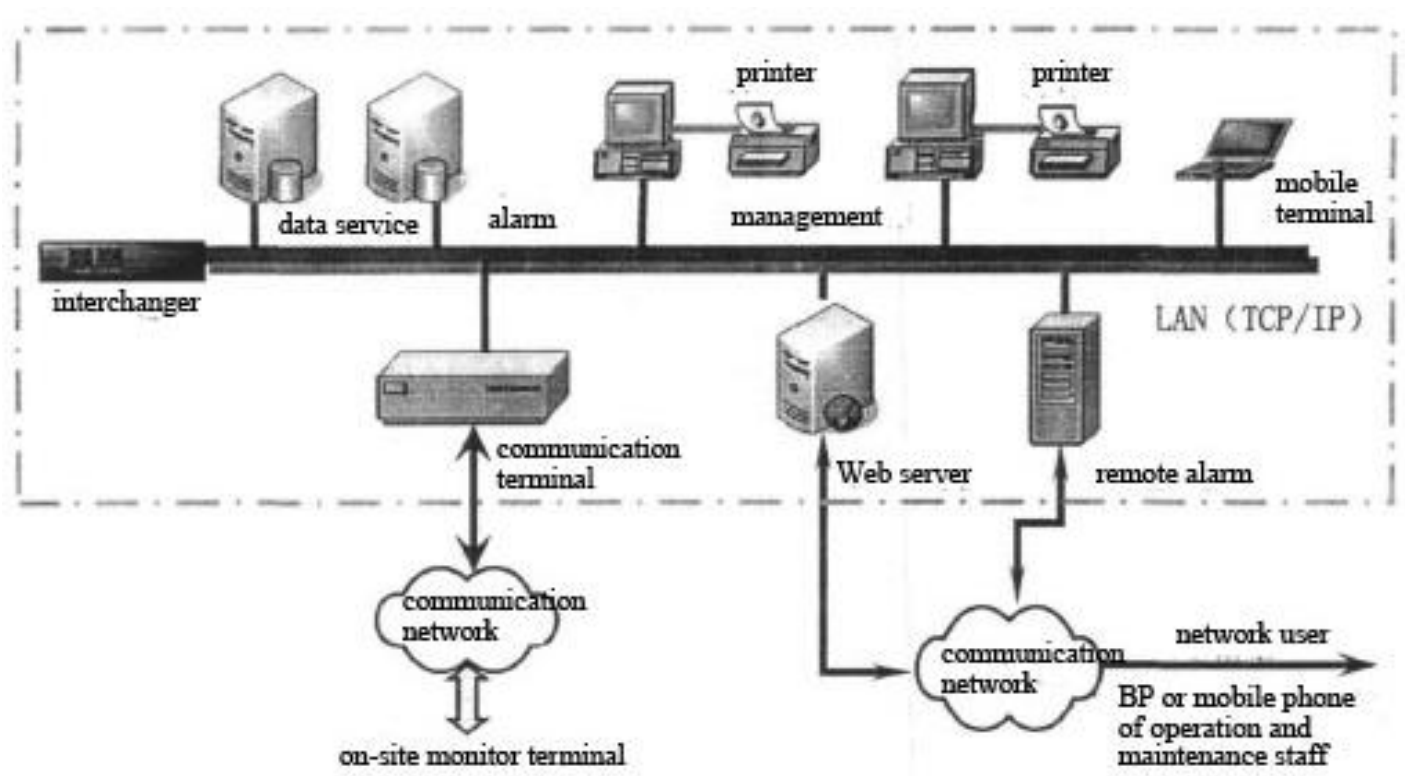

Figure 2. The working structure of the surveillance center 


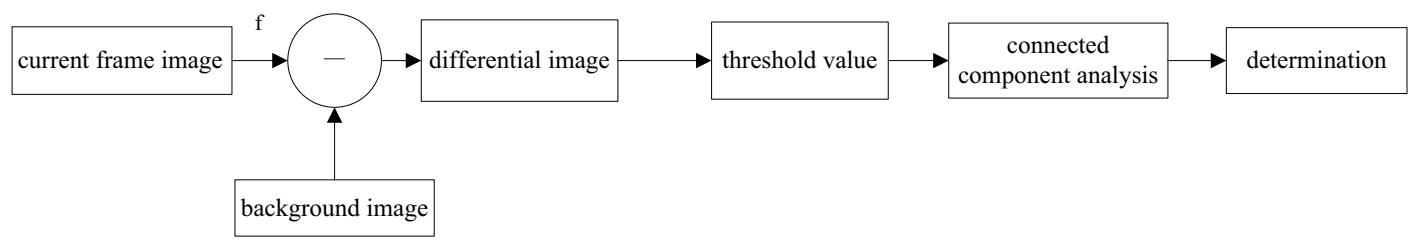

Figure 3. Operational process

Video surveillance system mainly consists of three parts, namely remote substation, centralized control station and surveillance center (master control center). The responsibility of the surveillance center is to conduct unified regulation on the video surveillance, the network and videos of all devices and to authorize each centralized control center as well as its region. As for the surveillance center, the management interface includes two parts, the user configuration of high-level administrators and the configuration of the maintenance system. For the security, only administrators are permitted to log onto the control center with the privilege to set up a database ${ }^{[7]}$ so as to regulate the configuration of facilities and authorize clients. In addition, the operation log of any user is recorded in detail $^{[8]}$ so that operations are well documented. Operation records of this kind guarantee the safe operation of the system effectively.

\section{KEY TECHNOLOGIES OF THE PLATFORM}

Key technologies of the platform are the target characteristic detection method based on vision and the means of pattern classification, which screen and analyze a large amount of videos intelligently and realize accurate warnings of the work of unattended substations. The calculation and the function realization of the detection algorithm are introduced as follows:

\section{Detection algorithm}

As for the intelligent video analysis system of complicated fields like substations, the target characteristic detection method based on vision and the means of pattern classification are mainly adopted. Detecting steps are provided below:

\section{1) Background modeling of the field}

This platform carries out static field modeling with images and videos that are collected from the working site of a substation and updates the model built by the newly collected images in time ${ }^{[9]}$. Gaussian mixture model is adopted in this paper for modeling. Multiple probability distributions are needed to describe a field and realize background subtraction:

$$
\begin{aligned}
& \omega_{k, t}=(1-\alpha) \omega_{k, t}-1+\alpha\left(M_{k, t}\right) \\
& \mu_{t}=(1-\beta) \mu_{t-1}+\beta X_{t} \\
& \sigma_{t}^{2}=(1-\beta) \sigma_{t-1}^{2}+\beta\left(X_{t}-\mu_{t}\right) I\left(X_{t}-\mu_{t}\right)
\end{aligned}
$$

$$
\begin{aligned}
& M_{k, t}=\left\{\begin{array}{l}
1, \text { matched } \\
0, \text { unmatched }
\end{array}\right. \\
& \beta=\alpha \eta\left(\mathrm{X} \mid \mu_{k}, \sigma_{k}\right)
\end{aligned}
$$

Where, $\alpha$ is a constant term, representing the update rate of the Gaussian mixture model.

$\frac{1}{\alpha}$ stands for the change rate of the model parameters; $M_{k, t}$ is a constant term, which is defined in Formula (4); $\beta$ is an automatic adjusting term, standing for the learning rate of the Gaussian mixture model defined in Formula (5). It is worth noting that Formula (2) and Formula (3) update only for the matched Gaussian distribution while maintain parameters unchanged for other unmatched Gaussian distribution.

\section{2) Target detection}

After the acquisition of background information, the background subtraction is applied to detecting whether there are suspicious targets in newly obtained images. Lock possible suspicious targets so as to provide convenience for tracking and behavior analysis ${ }^{[10]}$. The operational process is shown in Figure 3.

In this figure, $f_{n}$ stands for the current frame image; $\mathrm{B}$ is the background image. $B(x, y)$ and $B(x, y)$ respectively stand for the background image and the gray value of the pixel of $(x, y)$ in the current frame image. Conduct the difference algorithm shown below and take the absolute value in the meantime so as to obtain the differential image Dn.

Set the threshold value $\mathrm{T}$ and carry out binary processing on the differential image. As shown in Formula (6), thresholds that are larger than this threshold are evaluated as 1, standing for moving target points; otherwise, thresholds are evaluated as $\left(R^{\prime}\right.$ anding for background points. The binary image $R_{n_{\text {with }} \text { sepa- }}$ rated target and background can be obtained thusly.

\section{3) Feature extraction}

This platform adopts the feature algorithm of Histogram of Oriented Gradient (HOG) for the feature detection of such devices as transformers and disconnecting switches in a substation. The detection on device status changes can be realized accurately through the combination of HOG feature algorithm and SVM classifier.

The HOG feature extraction algorithm used in this paper grays the detection target first (regarding the image as a $3 \mathrm{~d}$ image of $\mathrm{x}, \mathrm{y}, \mathrm{z}$ (gray scale)) and stand- 


\section{MATEC Web of Conferences}

ardizes (normalizes) the color space of the input image with Gamma correction method. The objective is to adjust the contrast of the image, reduce the impact brought by the local shadow and illumination changes of the image, and suppress the interference of noise The calculation of the gradient (including size and direction) of each image aims at mainly capturing the outline information and further reducing the interference of light. The image is divided into small cells (for example, $6 * 6 \mathrm{pixel} / \mathrm{cell})$; provide the gradient histogram of each cell (the number of different gradients) so as to form the descriptor of each cell; a block is formed by several cell (for example, $3 * 3$ cell/block) and the HOG feature descriptor of the block can be acquired through the connection of all the cell features in one block. The HOG feature descriptor of the image (the target of your detection) can be obtained through the connection of all HOG feature descriptors of the block in the image. This is the final eigenvector for the classified usage (formalized).

\section{4) Pattern classification}

Based on the database of data samples and feature extraction, trainings are conducted in SVM ${ }^{[11]}$. Linear SVM is adopted for training on account of the problem of speed. In this paper, SVM classifier is applied in the pattern classification of detection targets. As for two classifiers based on SVM and the SVM with poor classification accuracy, the weighted voting is conducted to the SVM classifier through AdaBoost algorithm so as to increase the classification viscosity. The training diagram of the algorithm is shown in Figure 4.

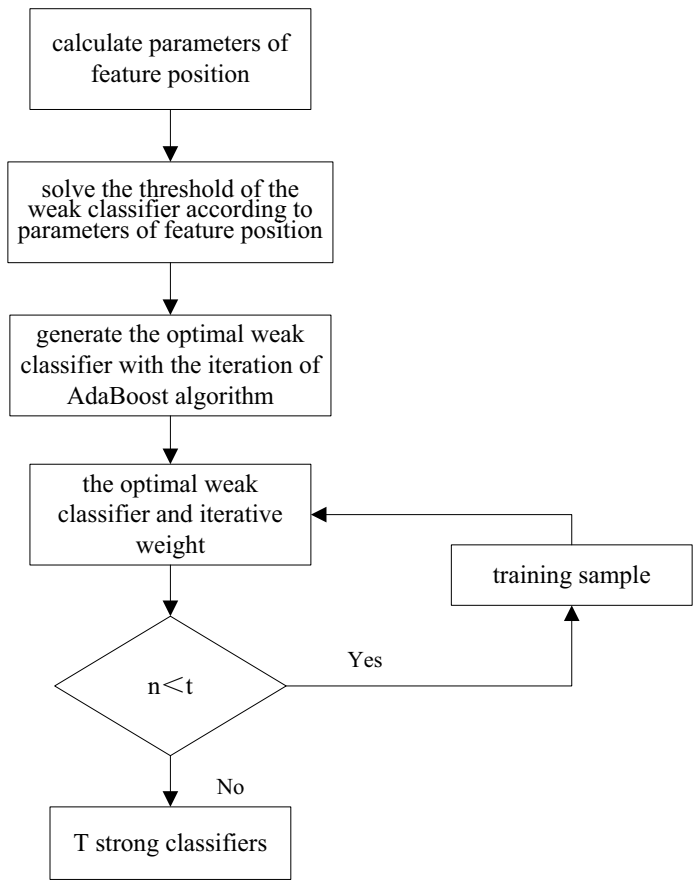

Figure 4. Training diagram of AdaBoost algorithm

The technology is able to realize effective analyses on surveillance videos and data of substations as well as on intrusion detection, foreign substance detection, fire and smoke detection and oil leakage detection. The algorithm has a strong robustness, the system structure of which is able to carry out real-time detections on video information and interact with the technology supporting system in analyses and applications As for regulation and surveillance staffs, the monitoring efficiency and means of remote failure judgment are both increased largely.

\section{FUNCTION REALIZATION OF THE PLAT- FORM}

This platform establishes a multimedia database of typical scenes. This database is a comprehensive multimedia database, including not only equipment scenes, background data, abnormal incident data (like the data of transformer oil leakage), personnel intrusion behavior data (staying, wandering, and crossing) but also the understanding of scenes and pre-defined parameters. After the acquisition of background information on this basis, the target characteristic detection method and the means of pattern classification are adopted to realize the automatic identification and monitoring of the key mapping state changes of substation equipment, realize the automatic identification and warning of safe working behaviors in the complex background, and further enrich methods of routing inspection, equipment failure detection, maintenance means, abnormal behavior identification as well as methods of intelligent retrieval of multimedia data. With the processing of intelligence analysis, equipment hidden dangers brought by security protection, defense and bad weather of a substation and remnants around the substation can be found in the first time. The comparison diagram of the traditional surveillance model and the intelligent surveillance model is provided below in table 1 .

\section{SUMMARY AND OUTLOOK}

With the help of the powerful video data processing function, the platform carries out high-speed analyses on video data through different algorithms, filters out uninteresting information of users and emphasizes valuable information for surveillance staff, reducing the labor intensity of video surveillance staff as well as false and missing alarms significantly and increasing the efficiency of processing alarm events. Meanwhile, the extraction of key information can lead to applications with high values through the combination with other grid surveillance systems.

With the development of high-definition video technology and the constant enrichment of network resources, high-definition videos and the existing standard definition videos will be unified in the same platform and surveillance methods of the platform will become more refined. Considering more special factors, opinions can be sought in a larger range in terms 
ICETA 2015

Table1.The comparison diagram of the traditional surveillance model and the intelligent surveillance model

\begin{tabular}{|c|c|c|}
\hline surveillance model & $\begin{array}{l}\text { traditional surveillance } \\
\text { model }\end{array}$ & intelligent surveillance model \\
\hline $\begin{array}{l}\text { regulate and monitor cycles of major parameters of grid } \\
\text { operation }\end{array}$ & $120 \mathrm{~min}$ & $4 \sim 5 \mathrm{~min}$ \\
\hline $\begin{array}{l}\text { out-of-limit monitoring cycle of equipment overload, oil } \\
\text { temperature, voltage and power factor }\end{array}$ & $120 \mathrm{~min}$ & $1 \sim 2 \mathrm{~min}$ \\
\hline monitoring intellectualization of power net tide control limit & $\begin{array}{l}\text { artificial calculation has } \\
\text { fault surfaces and dif- } \\
\text { ficulties in surveillance }\end{array}$ & $\begin{array}{l}\text { automatic calculation and } \\
\text { classified comparison in line } \\
\text { with preset values }\end{array}$ \\
\hline out-of-limit automatic sequence & - & $\begin{array}{l}\text { alarm according to the auto- } \\
\text { matic sequence of the fault } \\
\text { surface of power grid equip- } \\
\text { ment }\end{array}$ \\
\hline critical out-of-limit state & - & $\begin{array}{l}99 \% \text { discovery rate, auto- } \\
\text { matic hint, instant screen } \\
\text { push }\end{array}$ \\
\hline man-machine conversation & - & $\begin{array}{l}\text { reserved interface, the moni- } \\
\text { toring range of pre-warning } \\
\text { is automatically determined } \\
\text { according to equipment } \\
\text { working conditions }\end{array}$ \\
\hline
\end{tabular}

of evaluation indicators so that indicators are able to meet the requirements of video surveillance in substations.

\section{REFERENCES}

[1] Jing, M.L. 2012. A Study on the Intelligent Video Surveillance Technology of Unattended Substations, [Master's degree], North China Electric Power University.

[2] Yang, S.J. 2001. A discussion on the design idea of unattended substations, Relay, (09): 68-70.

[3] Liu, X.Z., Lou, J.X., Zheng, Q.F., et al. (2010). Behavior function design and implementation of the substation video surveillance system, Automation of Electric Power Systems.

[4] Li, T.F. 2007. Schematic Design of the Substation Video Surveillance System, Tianjin: Tianjin University.

[5] Chen, W.S., Lin, Y.F., Tan, H., et al. 2004. The application of substation remote digital video surveillance technology, East China Electric Power, 5: 39-41.

[6] Kamarulafizam, I., Sh-Hussain., Mokji, M.M., Ronisham, S., Ghani, A. \& Yusof, M. 2005. Time-frequency analysis of power signal: Application to substation monitoring and management system. Computers Communications \& Signal Processing with Special Track on Biomedical Engineering.

[7] Jiang, Q.H. 2010. Online monitoring system of electric transmission line and its application, Hubei Electric Power, 34(Add): 84-85.

[8] Yaniv Gurwicz, Raanan Yehezkel. \& Boaz Lachover, 2011. Multiclass object classification for real-time video surveillance systems, Pattern Recognition Letters.

[9] R Moreno, M GraiTa \& E Zulueta. 2010. RGB color gradient following color constancy preservation, Electronics Letters, 46(13): 908-910.
[10]Naeem Ramzan, Toni Zgaljic, Ebroul Izquierdo. An efficient optimization scheme for scalable surveillance centric video communications, Signal Processing.

[11]Wann-Yun Shieh \& Ju-Chin Huang. 2012. Falling-incident detection and throughput enhancement in a multi-camera video-surveillance system, Medical Engineering \& Physics. 